\title{
Reinigung auf kleiner Flamme
}

\section{FREIER KOPF durch Unterdruck, Entspannung und ätherische Öle \\ Carola Texter-Bögeholz}

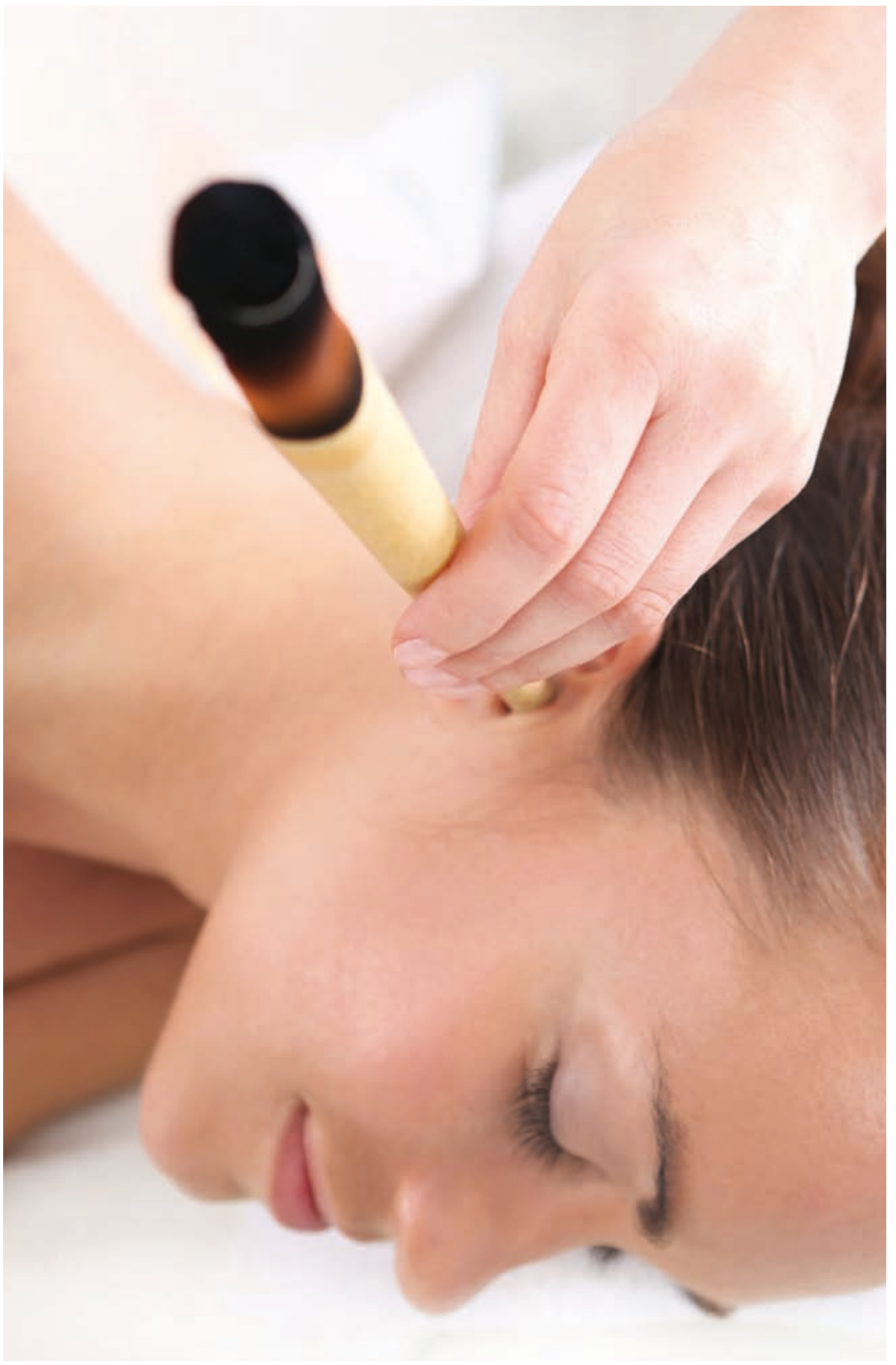

DIE VERWENDUNG von Ohr- oder Hopi-Kerzen ist sowohl aus der traditionellen indianischen Heilkunde Nordamerikas als auch aus der indonesischen Naturmedizin überliefert und diente ursprünglich der Reinigung, Stärkung, Harmonisierung sowie zu rituellen Zwecken. Frühe Hinweise finden sich im 12. Jahrhundert. Fast in Vergessenheit geraten, erfuhren Ohrkerzen ab etwa 1990 eine Renaissance, wobei sich das Indikationsfeld deutlich erweiterte. Sie werden namensgemäß meist im Ohr aufgesetzt, können jedoch auch für alle anderen Körperbereiche verwendet werden, dann unter der Bezeichnung Körperkerzen.

Ohrkerzen werden gewöhnlich fertig aus dem Fachhandel bezogen. Zur Herstellung wird aus traditionellen Materialien wie Gaze, Bienenwachs und ätherischen Ölen ein ca. 20-30 cm langes, zylindrisches oder konisch (trichterförmig) zulaufendes Rohr von etwa $1 \mathrm{~cm}$ Durchmesser gefertigt, häufig unter Zugabe heilender Pflanzenextrakte oder -pulver.

\section{Feuer der Entlastung: entstauend, wärmend, anregend}

Durch die im Zuge der Verbrennung erwärmte Luft stellt sich im Inneren der Ohrkerze ein starker Luftstrom ein, man spricht von Kamineffekt. Somit entsteht am unteren Ende der Kerze (diese muss den Gehörgang dicht abschließen) bis hin zum Trommelfell eine Sogwirkung mit Unterdruck. Dies entlastet Trommelfell, Mittel- und Innenohr, ohrnahe Lymphbahnen, die Eustachi-Röhre (s. S. 28) bis hin zu den Nebenhöhlen, den Augen und der Rachenmandel. Stauungen können sich lösen, die Druckregulation und der Augeninnendruck verbessern sich, häufig auch das Hören. Auch der Gehörgang wird gereinigt.

Der Patient spürt evtl. eine (erwünschte) angenehme Wärme oder Hitze, hört das entspannende Knistern der abbrennenden Ohrkerze und nimmt eine wohltuende Druckentlastung und evtl. einen entlastenden Sekretfluss im Bereich der Nasenhöhle wahr. Die Augen können dabei tränen. 
Auch die verschwelenden Gewürze und ätherischen Öle sowie eine angenehme Umgebung und Atmosphäre tragen zum Gesamteffekt bei. Daher eignet sich die Ohrkerzentherapie hervorragend als ergänzende Regulations- oder Reiztherapie in der Naturheilpraxis.

Da Ohrkerzen sehr gut verträglich sind und bei sachkundiger Anwendung kaum Nebenwirkungen hervorrufen, lassen Sie sich grundsätzlich bei allen Patienten ab etwa 5 Jahren anwenden, bei denen eine ruhige Lagerung über die Behandlungszeit beschwerdefrei möglich ist. Gerade Kinder sprechen gut auf die entspannende, entlastende Wirkung an.

\section{Indikationen: Ohrbeschwerden, Stress, Infekte}

$\mathrm{Zu}$ den wichtigsten Indikationen der Ohrkerzentherapie zählen:

- Ohrenschmerzen (nicht akut entzündlich)

- grippale Infekte, „Verschleimungen“ jeglicher Art im Kopf

- Entschlackung

- Husten bis zur beginnenden Bronchitis

- (beginnende) Sinusitis im Bereich der Stirnoder Kieferhöhlen

- Migräne, Spannungskopfschmerz (auch bei Kindern)

- Tinnitus

- begleitend bei Hörsturz

- sonstige Hörstörungen

- Druckgefühl im Auge

- nervöses Zucken der Augenlieder

- Stresserkrankung, Spannungszustände, Schlafstörungen

- Schwindel

- Hypertonie

- Pollenallergie

Patienten genießen nicht nur die Wirkung der Ohrkerze, sondern auch die Zuwendung des Therapeuten und ihre Auszeit: Andere Tätigkeiten können und müssen während der Behandlung nicht ausgeführt werden.

\section{Nebenwirkungen: \\ Sichergehen mit Einschleichen}

Nebenwirkungen treten im Rahmen der Ohrkerzentherapie nur in seltenen Fällen auf, darunter leichte Formen von Schwindel, Zahnschmerzen und Fieber sowie Tränenfluss und Hautreizungen. Im Regelfall klingen die Symptome innerhalb kurzer Zeit ab. Um unvorhergesehene Nebenwirkungen zusätzlich so gering wie möglich zu halten, kann die Therapie über 2-3 Sitzungen eingeschlichen werden.

\section{Inhaltsstoffe: Mit ätherischen Ölen die Wirkung potenzieren}

Meist dienen Honig und Bienenwachs mit ihren harmonisierenden und reinigenden Eigenschaften als Zusätze. Darüber hinaus lässt sich die therapeutische Wirkung durch den Zusatz ätherischer Öle - wenn diese gut vertragen werden - hervorragend beeinflussen und somit die Ohrkerzentherapie mit der Aromatherapie kombinieren (s. Tabelle 1 ).

\section{KURZ GEFASST}

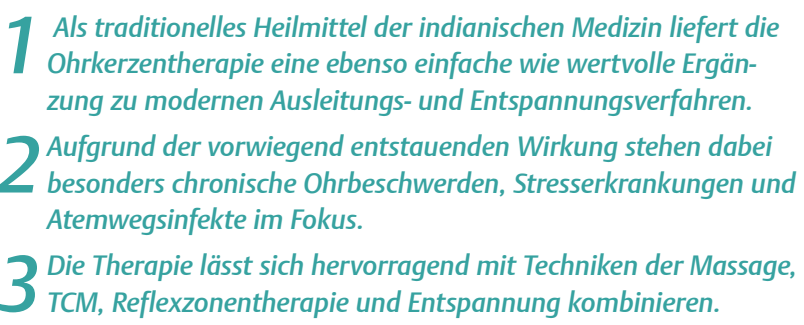

\section{Körperkerzen: Blockaden lösen, Reflexzonen aktivieren}

Werden Ohrkerzen als Körperkerzen in anderen Körperbereichen aufgesetzt, erzielen sie dort vorwiegend eine durchblutungsfördernde, entschlackende, energetisierende und deblockierende Wirkung, weshalb sie gerne verwendet werden, um Blockaden zu lösen. Sie lassen sich je nach Indikation u. a. an Reflexzonen, Akupunkturpunkten, Akupressurfeldern, Head-Zonen, in Narbengebieten oder anderen Störfeldern sowie den Chakrenpunkten aufsetzen. Entsprechend des Applikationsorts und der jeweiligen Zusätze lässt sich somit eine aktivierende, sedierende, entstörende oder harmonisierende Wirkung erreichen. Die Blockaden werden auf körperlicher, seelischer und geistiger Ebene gelöst.

\section{Praktische Anwendung in 5 Schritten}

Die Anwendung der Ohrkerzentherapie lässt sich in 5 Schritte gliedern.

\section{Vorbereitung}

Für die Anwendung benötigen Sie 2 Ohrkerzen mit passendem Durchmesser (je nach Querschnitt des Gehörgangs 5, 6 oder 7 mm) und der Indikation entsprechenden Zusätzen (s. Tabelle 1), außer-

\section{INFORMATION}

\section{Kontraindiziert ist die Ohrkerzen- therapie bei:}

- Kindern unter 3 Jahren

- Psychosen

- Allergien auf Inhaltsstoffe der Ohrkerzen

- akuten Entzündungen und/oder Verletzungen im Bereich von äußerem Gehörgang, Mittel- oder Innenohr (z. B. Otitis media)

- schweren Erkrankungen des Mittelohrs

- Perforationen des Trommelfels

- Ohrimplantaten

- Pilzinfektionen des äußeren Gehörgangs

- Fieber 
dem die beigefügten Schutzscheiben (behelfsweise Aluminiumfolie), ein Feuerzeug sowie einen kleinen Behälter mit Wasser zum Auslöschen der Kerzen nach der Behandlung. Für die Körperkerzenanwendung ist außerdem eine Kompresse zum Abdecken der Haut erforderlich. Auch ein Getränk für den Patienten sollte bereitstehen: Trinken vor und nach der Behandlung unterstützt die Entgiftung. Der Raum sollte hinsichtlich Temperatur, Geruch, Geräuschen und Ambiente die Entspannung unterstützen.

\section{Wichtig: Beachten Sie beim Kauf, dass die Ohrkerzen gemäß EU-Richtlinie 93/42/EWG als Medizinprodukt ausgewiesen sind.}

\section{Lagerung und Abbrennschutz}

Für die Anwendung den Patienten bequem auf der Seite lagern, sodass der Gehörgang senkrecht nach oben weist, den Kopf durch ein Kissen oder eine Rolle unterstützen. Auch Knierollen und De- cken (für fröstelnde Patienten) können hilfreich sein. Haare und Kleidung aus dem Behandlungsbereich entfernen. Nun die beigefügte Schutzscheibe über das schmalere Ende der Kerze bis zur Markierung ziehen. Diese schützt nicht nur Ohr und Gesicht des Patienten, sondern auch die Hand des Therapeuten vor Verbrennungen, welche sehr selten und insbesondere durch herabtropfendes Wachs auftreten.

\section{Ohrmassage, Entzünden und Einführen}

Eine sanfte Ohrmassage zu Behandlungsbeginn sorgt für Entspannung und eine bessere Durchblutung. Dabei fallen auch mögliche Entzündungen, Verhärtungen, Verletzungen oder anatomische Besonderheiten auf. Die Ohrkerze nun oben (am breiteren Schaftende) entzünden, sodass eine Flamme entsteht, evtl. mit feiner Rauchbildung. Mit einer sanften Drehbewegung daraufhin das schmalere, nicht brennende Ende langsam und ohne Druck senkrecht in das freiliegende Ohr einführen, bis es den äu-

\begin{tabular}{|c|c|}
\hline \multicolumn{2}{|c|}{ Ätherische Öle als Zusätze in Ohrkerzen und ihre Wirkungen } \\
\hline Zusatz & Wirkung \\
\hline Bitterorange & beruhigend, stimmungsaufhellend \\
\hline Eukalyptus & $\begin{array}{l}\text { auswurffördernd, antimikrobiell, antiphlogistisch, schmerzlindernd, } \\
\text { krampflösend, heilungsfördernd, reinigend }\end{array}$ \\
\hline Kamille & $\begin{array}{l}\text { entkrampfend, beruhigend, antiphlogistisch, schmerzlindernd, } \\
\text { heilungsfördernd }\end{array}$ \\
\hline Kiefernadeln & antiphlogistisch, antimikrobiell, durchblutungsfördernd, tonisierend \\
\hline Lavendel & $\begin{array}{l}\text { entkrampfend, emotional harmonisierend, entspannend, schlaf- } \\
\text { fördernd, entzündungshemmend, stimmungsaufhellend, schmerz- } \\
\text { lindernd, antibakteriell, heilungsfördernd }\end{array}$ \\
\hline Lemongras & $\begin{array}{l}\text { stimmungsaufhellend, anregend, antiphlogistisch, gefäßerweiternd, } \\
\text { keimwidrig, immunstimulierend }\end{array}$ \\
\hline Pfefferminze & $\begin{array}{l}\text { erfrischend, kühlend, schmerzlindernd, antimikrobiell, krampflösend, } \\
\text { konzentrationsfördernd, nervenstärkend, kräftigend }\end{array}$ \\
\hline Rosmarin & $\begin{array}{l}\text { anregend, tonisierend, schleimlösend, durchblutungsfördernd, } \\
\text { entkrampfend, schmerzlindernd, antimikrobiell }\end{array}$ \\
\hline Salbei & kräftigend, adstringierend, antiphlogistisch, vitalisierend \\
\hline Teebaum & $\begin{array}{l}\text { antimikrobiell, antiphlogistisch, abschwellend, schmerz- und juckreiz- } \\
\text { lindernd, nervenstärkend, immunstimulierend, erfrischend }\end{array}$ \\
\hline Ylang-Ylang & antidepressiv, beruhigend, motivierend \\
\hline
\end{tabular}


ßeren Gehörgang luftdicht verschließt, sodass kein Rauch (mehr) aus dem unteren Kerzenbereich aufsteigt. Die Kerze soll während der Behandlungsdauer angenehm, schmerzfrei und senkrecht im Ohr sitzen. Das entzündete Ende der Kerze glimmt bei der Nutzung nur noch.

\section{Abbrennen der Ohrkerze}

Obwohl die Ohrkerze i. d. R. sicher im Gehörgang fixiert ist, hält sie die Hand des Therapeuten während des gesamten Abbrennens (dabei hinter dem Ohr leicht auflegen), welches ca. 1015 min dauert. Die Schutzscheibe bleibt weiterhin um die Kerze fixiert.

\section{Abnehmen und Nachbehandlung}

Kerze mit leichter Drehbewegung aus dem Ohr des Patienten entfernen, wenn sie bis zur Markierung abgebrannt ist, im dafür bereitgestellten Wasserbehälter löschen und entsorgen. Nun den äußeren Gehörgang des Patienten auf Rückstände, Rötungen etc. kontrollieren und mit Öl oder Creme behandeln, z. B. Körperöl (ohne Paraffin).

Nach Umlagerung des Patienten auf die andere Seite auch das andere Ohr wie beschrieben behandeln: Für eine ausgewogene Wirkung werden immer beide Ohren behandelt.

Nach der Behandlung dem Patienten ein Getränk reichen und ihn 15-30 min (bei Hypotonie 30-45 min) nachruhen lassen. Somit dauert eine Sitzung etwa 40-50 min. Pro Indikation empfehlen sich bis zu 12-15 Behandlungen, 1- bis 2-mal pro Woche.

Kundige Patienten können Ohrkerzen nach Einweisung auch in der Selbstbehandlung anwenden, aufgrund der schlaffördernden und entspannenden Wirkung insbesondere abends vor dem Schlafengehen.

\section{Ohrenkerzen als Körperkerzen}

Werden Ohrkerzen als Körperkerzen verwendet, wird wie beschrieben vorgegangen und jede Kerze ca. 10 min manuell auf den Behandlungspunkt aufgesetzt (z. B. Head-Zone, Reflexzone, Akupressurfeld). Für weitere Behandlungen am Körper werden jeweils neue Kerzen entzündet.

Merke: Wird die Ohrkerze als Körperkerze verwendet, sollte die Haut durch eine aufgelegte Mullkompresse zwischen Kerze und Haut vor Hitze und Irritationen geschützt werden.

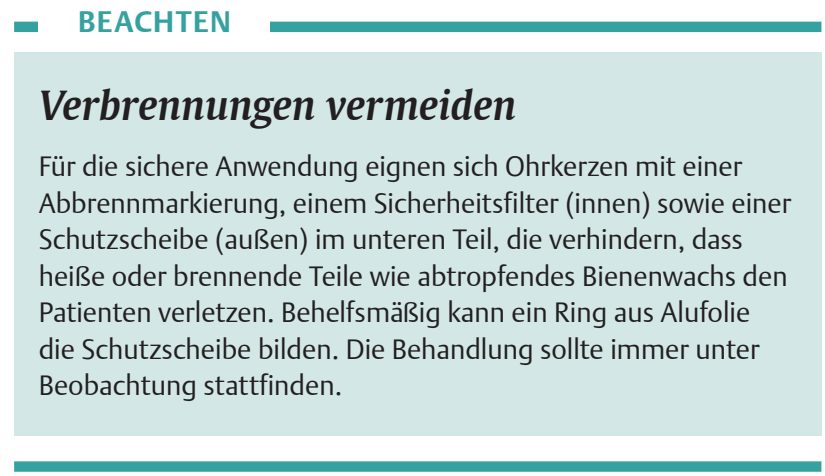

Als sanft regulatorische, reinigende und nebenwirkungsarme Behandlung lässt sich die Ohrkerzentherapie sehr gut mit anderen Therapien kombinieren, darunter Lymphdrainage am Kopf (Schädel, Gesicht, Hals), Fußreflexzonen- oder Ohrmassage, klassische Massage, Tuina, Akupressur, Akupunktur, Autogenes Training, Progressive Muskelentspannung, Achtsamkeitsübungen oder Hypnose.

Dieser Artikel ist online zu finden:

http://dx.doi.org/10.1055/s-0035-1563584

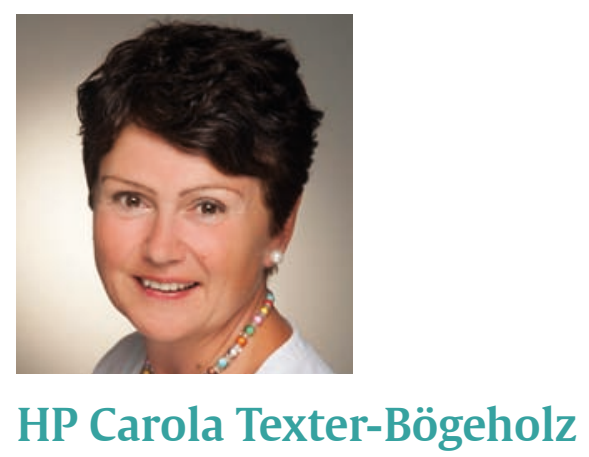

Friedrich-Ebert-Str. 37

32760 Detmold

E-Mail: carola-texter-boegeholz@t-online.de

Carola Texter-Bögeholz ist seit über 16 Jahren als Heilpraktikerin in Detmold tätig. Ihr Praxisschwerpunkt liegt in der Entspannungstherapie und dem Coaching mit Hypnose. Sie ist zudem seit vielen Jahren als Dozentin in Heilpraktikerschulen tätig. 